\title{
The TeV gamma-ray source population of the Milky-Way
}

\section{Vecchiotti, ${ }^{a, b, *}$ M. Cataldo, ${ }^{c}$ G. Pagliaroli ${ }^{a, b}$ and F.L. Villante ${ }^{b, d}$}

${ }^{a}$ Gran Sasso Science Institute,

67100 L'Aquila, Italy

${ }^{b}$ INFN, Laboratori Nazionali del Gran Sasso, 67100 Assergi (AQ), Italy

${ }^{c}$ Friedrich-Alexander-Univeristät Erlangen-Nürnberg, Erlangen Center for Astroparticle Physics, 91058 Erlangen, Germany

${ }^{d}$ University of L'Aquila, Physics and Chemistry Department,

67100 L'Aquila, Italy

E-mail: vittoria.vecchiotti@gssi.it

In this work we perform a population study of the H.E.S.S. Galactic Plane Survey (HGPS) catalogue. Namely, we analyze the flux, latitude and longitude distributions of gamma-ray sources detected by H.E.S.S. with the goal of inferring the main properties of galactic TeV sources population.

We show that the total Milky Way luminosity in the 1-100 TeV energy range is relatively well constrained by H.E.S.S. data, obtaining $L_{\mathrm{MW}}=1.7_{-0.4}^{+0.5} \times 10^{37} \mathrm{ergs} \mathrm{s}^{-1}$. The total Galactic flux in the H.E.S.S. observational window is $\Phi_{\text {tot }}=3.8_{-1.0}^{+1.0} \times 10^{-10} \mathrm{~cm}^{-2} \mathrm{~s}^{-1}$.

The above results allows us to estimate the flux produced by sources not resolved by H.E.S.S. These sources, which are too faint (or too extended) to be detected by H.E.S.S., contribute to the largescale diffuse signal observed at the $\mathrm{TeV}$ range. We show that unresolved source contribution is not negligible (about $60 \%$ of the resolved signal measured by H.E.S.S.) and potentially responsible for a large fraction of the diffuse-large scale gamma-ray signal observed by H.E.S.S. and other experiments in the $\mathrm{TeV}$ domain.

Finally, in the hypothesis that the majority of bright sources detected by H.E.S.S. are powered by pulsar activity, like e.g. Pulsar Wind Nebulae or TeV halos, we estimate the main properties of the pulsar population: we obtain a constrain on the fading time $\tau$, the initial period $P_{0}$ and the magnetic field $B$.

$37^{\text {th }}$ International Cosmic Ray Conference (ICRC 2021)

July 12 th - 23rd, 2021

Online - Berlin, Germany

\footnotetext{
${ }^{*}$ Presenter
} 


\section{Introduction}

The field of $\mathrm{TeV}$ astronomy is rapidly evolving thanks to the data obtained by recent experiments $[1,3]$. However, we still don't know which (if any) class of sources dominate Galactic emission and we still miss a robust determination of the diffuse $\gamma$-ray flux produced at $\mathrm{TeV}$ energies by cosmicray $(\mathrm{CR})$ interactions with the gas contained in the Galactic disk. The relatively large diffuse flux measured at $\mathrm{TeV}$ by Milagro, H.E.S.S. and HAWC could be explained either as the cumulative contribution of unresolved sources, or by considering non conventional CR propagation models characterised by position-dependent transport properties. In this work we perform a population study of the H.E.S.S. Galactic Plane Survey (HGPS) catalogue with the goal of inferring general property of the $\mathrm{TeV}$ sky that can be useful for future experiments and for disentangle the $\gamma$-ray diffuse emission from the unresolved sources component in this energy range. In addition we consider the regime where all bright sources observed by H.E.S.S. (which are not firmly identified as SNRs) are powered by pulsar activity, e.g. PWNe and/or TeV halos as suggested by [12] and we discuss the constraints on the pulsar properties, namely the initial spin-down period and the neutron star magnetic field, that are obtained by HGPS data.

\section{HGPS}

The H.E.S.S. Galactic Plane Survey (HGPS) catalogue [1] includes 78 VHE sources observed in the longitude range $-110^{\circ} \leq l \leq 60^{\circ}$ and for latitudes $|b|<3^{\circ}(\sim 80 \%$ of the Galactic plane), measured with an angular resolution of $0.08^{\circ}$ and a sensitivity $\simeq 1.5 \%$ Crab flux for point-like objects. It provides the integral flux above $1 \mathrm{TeV}$ of each source that is obtained assuming a power-law spectrum with index $\beta=2.3$.

In order to avoid selection effects, we restrict our analysis to the brightest sources that produce an integral flux larger than $10 \%$ of that produced by the CRAB nebula $\phi_{\mathrm{CRAB}}=2.26 \times 10^{-11} \mathrm{~cm}^{-2} \mathrm{~s}^{-1}$. Above this threshold, the catalogue can be considered complete (no contribution from unresolved sources) [1] and consists of 32 sources: 19 are unidentified, 3 are firmly associated with SNRs (Vela Junior, RCW 86, RX J1713.7-3946), 2 are objects showing evidence of both shell and nebular emission which we refer to as composite objects, and 8 are associated with PWN. This allows us to perform our analysis in full generality without being forced to hypothesize a prescribed physical dimension for the sources because the angular extension does not discriminate the possible identification. A possible exception is provided by very close and very extended sources that cover angular regions larger than $\sim 1^{\circ}$ and could escape detection due to background subtraction procedure employed by H.E.S.S. We checked, however, that this situation is unlikely and, thus, does not affect our constraints unless one assumes that the majority of the observed sources have physical extension much larger than $f e w \times 10 \mathrm{pc}$.

\section{Method}

In order to predict the signal observed by H.E.S.S., we need to consider the spatial and intrinsic luminosity distribution of the $\mathrm{TeV}$ sources. We assume that this can be factorized as the product:

$$
\frac{d N}{d^{3} r d L}=\rho(\mathbf{r}) Y(L)
$$


where $\mathbf{r}$ indicates the distance from the Galactic center and $L$ is the $\gamma$-ray luminosity integrated in the energy range $1-100 \mathrm{TeV}$ probed by H.E.S.S.. The function $\rho(\mathbf{r})$, which is conventionally normalized to one when integrated in the entire Galaxy, is assumed to be proportional to the pulsar distribution in the Galactic plane parametrized by [10]. The source density along the direction perpendicular to the Galactic plane is assumed to scale as $\exp (-|z| / H)$ where $H=0.2 \mathrm{kpc}$ represents the thickness of the Galactic disk.

We assume that the intrinsic luminosity distribution $Y(L)$ can be parameterized as a power-law:

$$
Y(L)=\frac{\mathcal{N}}{L_{\max }}\left(\frac{L}{L_{\max }}\right)^{-\alpha}
$$

that extends in the luminosity range $L_{\min } \leq L \leq L_{\max }[11]$.

The parameter $\mathcal{N}$ defined in Eq. (2) determines the high-luminosity normalization of the function $Y(L)$; it represents the number of sources per logarithmic luminosity interval at the maximal luminosity (i.e. $d N / d \ln L=\mathcal{N}$ for $L=L_{\text {max }}$ ).

The above distribution can be naturally obtained by assuming a population powered by pulsar activity for which the intrinsic luminosity is proportional to the spin-down power and can be written:

$$
L(t)=\lambda(t) \dot{E}(t)=L_{\max }\left(1+\frac{t}{\tau_{\mathrm{sd}}}\right)^{-\gamma}
$$

where $\dot{E}$ is the spin-down power:

$$
\dot{E}=\dot{E}_{0}\left(1+\frac{t}{\tau_{\mathrm{sd}}}\right)^{-2}
$$

for energy loss dominated by magnetic dipole radiation (braking index $n=3$ ), $\lambda(t) \leq 1$ represents the efficiency of $\mathrm{TeV}$ emission and it is assumed to scale with time according to $\lambda(t)=\lambda\left(\dot{E} / \dot{E}_{0}\right)^{\delta}$ as suggested by [2] and $L_{\max }=\lambda \dot{E}_{0}$ is the initial luminosity. Therefore $\gamma=2(\delta+1)$. In our work we assume as reference case the one in which $\delta=0$, hence $\lambda(t)$ does not depend on time and $\gamma=2$, that corresponds to a source luminosity function $Y(L) \propto L^{-1.51}$. Assuming that the birth-rate $R$ of these sources in the Galaxy is constant in time then the Eq. 2 can be easily derived from Eq. 3. In this case $\alpha=1 / \gamma+1$ and the normalization $\mathcal{N}=R \tau_{\mathrm{sd}}(\alpha-1)$ represents the total number of young sources in the Galaxy that had not enough time to loose their initial luminosity and that are expected to be more easily detected by H.E.S.S.

The last necessary ingredient to predict the expected signal in H.E.S.S. is the relationship between the intrinsic luminosity $L$ of sources and the flux produced at Earth, that can be generally written as:

$$
\Phi=\frac{L}{4 \pi r^{2}\langle E\rangle}
$$

where $r$ is the source distance from the Galactic centre and $\langle E\rangle$ is the average energy of photons emitted in the range $1-100 \mathrm{TeV}$. In this work, we consider the average spectrum observed by HESS as a reference [1], i.e. we assume that all sources can be described by a power-law in energy with spectral index $\beta=2.3$ that corresponds to $\langle E\rangle=3.25 \mathrm{TeV}$.

\footnotetext{
${ }^{1}$ We also check in [5] the case in which $1+\delta=0.59 \pm 0.21$, as suggested by [2]. For this particular value of $\delta$, we obtain $\gamma \simeq 1.2$ in Eq.(3) that corresponds to a source luminosity function $Y(L) \propto L^{-1.8}$
} 
Using all the above ingredients we are able to determine the maximal luminosity $L_{\max }$ and the normalization $\mathcal{N}$ ( or the spin-down time scale $\tau_{s d}$ ) of the luminosity function by fitting H.E.S.S. observational results with an unbinned likelihood.

\subsection{Total flux and Milky Way luminosity.}

The determination of $L_{\max }$ and $\mathcal{N}$ allows us to estimate the total $\mathrm{TeV}$ luminosity produced by the considered population in the entire Galaxy which is given by:

$$
L^{\mathrm{MW}}=\frac{\mathcal{N} L_{\max }}{(2-\alpha)}\left[1-\Delta^{\alpha-2}\right]
$$

where $\Delta \equiv L_{\max } / L_{\min }$. The minimal luminosity $L_{\min }$ cannot be constrained by HESS observations. However, its value marginally affects the quantities considered in this paper, provided that $\Delta \gg 1$. Unless otherwise specified, we quote the results obtained for $\Delta \rightarrow \infty$ that can be easily scaled by using the above equation, if other values are considered.

By using Eqs. (1, 2, 5), it is also possible to calculate the flux at Earth produced by all sources (resolved and not resolved) included in the H.E.S.S. observational window (OW). This can be expressed as:

$$
\Phi_{\text {tot }}=\xi \frac{L_{\mathrm{MW}}}{4 \pi\langle E\rangle}\left\langle r^{-2}\right\rangle
$$

where the parameter $\xi$, which is defined as

$$
\xi \equiv \int_{\text {OW }} d^{3} r \rho(\mathbf{r})=0.812,
$$

represents the fraction of sources of the considered population which are included in the H.E.S.S. OW while the quantity $\left\langle r^{-2}\right\rangle$, defined as:

$$
\left\langle r^{-2}\right\rangle \equiv \frac{1}{\xi} \int_{\text {OW }} d^{3} r \rho(\mathbf{r}) r^{-2}=0.0176 \mathrm{kpc}^{-2}
$$

is the average value of their inverse square distance.

\subsection{Fading sources.}

The above description can be applied to potential TeV sources in the Galaxy, such as PWNe [8] or TeV Halos [9], which are connected with the explosion of core-collapse SN and the formation of a pulsar. The birth rate of these objects can be assumed proportional to that of SN explosions in our Galaxy, i.e. $R_{\mathrm{SN}}=0.019 \mathrm{yr}^{-1}$ as recently measured by [6]. The obtained best fit values for $L_{\max }$ and $\tau_{\text {sd }}$ can be linked to physical properties of the PWN namely the initial period $P_{0}$ and the neutron star magnetic field $B_{0}$ using [5]:

$$
\begin{aligned}
\frac{P_{0}}{1 \mathrm{~ms}} & =94\left(\frac{\lambda}{10^{-3}}\right)^{1 / 2}\left(\frac{\tau}{10^{4} \mathrm{yr}}\right)^{-1 / 2}\left(\frac{L_{\max }}{10^{34} \mathrm{erg} \mathrm{s}^{-1}}\right)^{-1 / 2} \\
\frac{B_{0}}{10^{12} \mathrm{G}} & =5.2\left(\frac{\lambda}{10^{-3}}\right)^{1 / 2}\left(\frac{\tau}{10^{4} \mathrm{yr}}\right)^{-1}\left(\frac{L_{\max }}{10^{34} \mathrm{erg} \mathrm{s}^{-1}}\right)^{-1 / 2}
\end{aligned}
$$



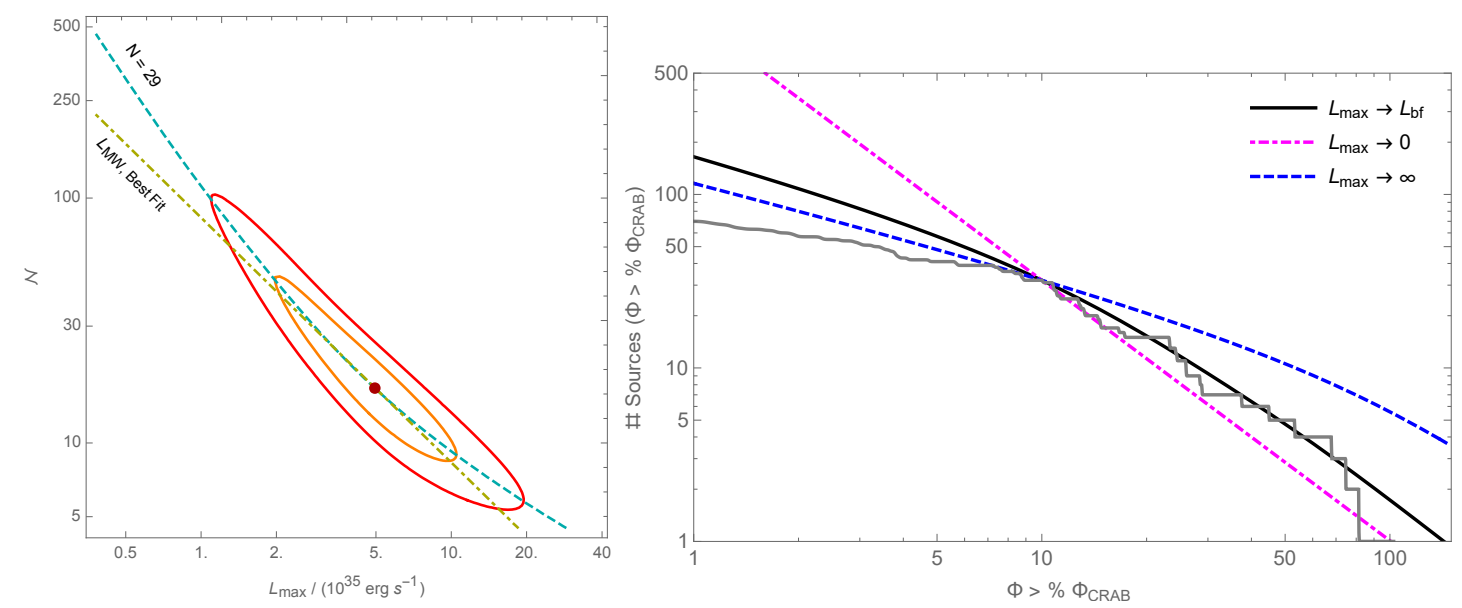

Figure 1: Left Panel: The best fit and the 1 and $2 \sigma$ allowed regions for $L_{\max }$ and $\mathcal{N}$. Right Panel: The cumulative distribution of the HGPS sources (gray line) compared with expectations for different values of the maximal luminosity $L_{\max }$.

provided that the fraction $\lambda$ of the spin-down power that is converted into $\mathrm{TeV} \gamma$-ray emission is known. The parameter $\lambda$ is highly uncertain; it is determined by the conversion of the spin-down energy into $e^{ \pm}$pairs and by the subsequent production of $\mathrm{TeV}$ photons. The values obtained for firmly identified PWNe in the HPGS catalogue fall between $5 \times 10^{-5}$ and $6 \times 10^{-2}$, see Tab.1 of [2]. For comparison, the value $\lambda \sim 3 \times 10^{-3}$ is obtained in [9] by studying the $\mathrm{TeV} \gamma$-ray emission of Geminga. In this work, we consider $\lambda$ as a free parameter, taking the value $\lambda=10^{-3}$ as a reference in numerical calculations.

\section{Results}

The best fit values and the allowed regions for the maximal luminosity $L_{\max }$ and the normalization $\mathcal{N}$ of the source luminosity distribution are shown in the left panel of Fig.1. We obtain $L_{\max }=$ $4.9_{-2.1}^{+3.0} \times 10^{35} \mathrm{ergs} / \mathrm{s}$ and $\mathcal{N}=17_{-6}^{+14}$ where the quoted uncertainties correspond to $1 \sigma$ confidence level (CL). The constraint on the maximal luminosity can be also expressed as $L_{\max }=13_{-6}^{+8} L_{\mathrm{CRAB}}$ by considering that the $\mathrm{CRAB}$ luminosity (above $1 \mathrm{TeV}$ ) is $L_{\mathrm{CRAB}}=3.8 \cdot 10^{34} \mathrm{ergs} / \mathrm{s}$. This results are obtained for our reference case with the power-law index of the luminosity distribution $\alpha=1.5$ and by neglecting the 3 HGPS sources which are firmly identified as SNRs, because they cannot be considered as fading sources. For a detailed discussion on the dependence and/or stability of the obtained results with respect to this and other assumptions in our analysis see Tab.1 in [5].

The obtained bounds are connected with specific features of the H.E.S.S. data. The constraint on the maximal luminosity essentially originates from the flux distribution of HGPS sources, as can be understood by looking at the right panel in Fig. 1 where we compare the cumulative number $N(\Phi)$ of observed sources with a flux larger than $\Phi$ with the predictions obtained for different $L_{\max }$ values. The theoretical calculations are normalized in such a way that the expected number of sources with $\Phi \geq 0.1 \Phi_{\mathrm{CRAB}}$ is equal to the observational value $N_{\mathrm{obs}}=29$. This corresponds to moving along the cyan dashed line in the left panel in Fig.1 that maximizes the likelihood for each assumed $L_{\max }$. The black line in the left panel in Fig.1 corresponds to the best fit value 
$L_{\max }=13 L_{\mathrm{CRAB}}$ and well reproduces the flux distribution in the range $\Phi \geq 0.1 \Phi_{\mathrm{CRAB}}$ considered in our analysis. For comparison, we also show the magenta dot-dashed line and the blue dotted line in the same figure that correspond to the limiting cases $L_{\max } \rightarrow 0$ and $L_{\max } \rightarrow \infty$, respectively. The limit $L_{\max } \rightarrow 0$ represents the case in which all sources have a very low luminosity, therefore, in order to be resolved by H.E.S.S., should be located in a small region surrounding the detector. The limit $L_{\max } \rightarrow \infty$, instead, corresponds to the possibility for the detector to investigate all the Milky Way within the OW. The cumulative distribution of sources observed by H.E.S.S. has a different behaviour with respect to both cases and thus it requires a specific $L_{\max }$ value in order to be reproduced. The possibility to determine $L_{\max }$ from the flux distribution automatically implies the ability to fit the normalization $\mathcal{N}$ of the source luminosity function by considering the additional constraint provided by the total number of observed sources (cyan dashed line in the left panel of Fig.1).

By using Eqs.(6) and (7), we obtain a determination of the total luminosity of the Galaxy in the energy range $1-100 \mathrm{TeV}$ and of the total flux (in the same energy range) produced by sources in the H.E.S.S. OW. We get: $L_{\mathrm{MW}}=1.7_{-0.4}^{+0.5} \times 10^{37} \mathrm{ergs} \mathrm{s}^{-1}$ and $\Phi_{\mathrm{tot}}=3.8_{-1.0}^{+1.0} \times 10^{-10} \mathrm{~cm}^{-2} \mathrm{~s}^{-1}$ that correspond to $L_{\mathrm{MW}}=445_{-112}^{+138} L_{\mathrm{CRAB}}$ and $\Phi_{\mathrm{tot}}=16.8_{-3.5}^{+4.4} \Phi_{\mathrm{CRAB}}$ in CRAB units.

The total flux at the Earth $\Phi_{\text {tot }}$ should be compared with the cumulative emission produced by all the 78 resolved sources in the HGPS catalogue, i.e. $\Phi_{\mathrm{HGPS}}=10.4 \Phi_{\mathrm{CRAB}}$. We obtain by subtraction the unresolved flux $\Phi_{\mathrm{NR}}=6.4_{-3.5}^{+4.4} \Phi_{\mathrm{CRAB}}$ which is due to sources in the considered population that are too faint to be identified by H.E.S.S.. We notice that the unresolved emission $\Phi_{\mathrm{NR}}$ is relatively large compared to the resolved source contribution $\Phi_{\mathrm{NR}} \simeq 60 \% \Phi_{\mathrm{HGPS}}$ (in agreement with our previous estimate in [4]) and also compared to the total flux in the H.E.S.S. OW (resolved+unresolved) $\Phi_{\mathrm{NR}} \simeq 38 \% \Phi_{\text {tot }}$. In conclusion, our results show that unresolved sources are likely to provide a relevant contribution to the diffuse large-scale $\gamma$-ray signal observed by H.E.S.S. and other experiments, with profound implications for the interpretation of observational results in the $\mathrm{TeV}$ domain.

\subsection{Interpretation in terms of a fading source population}

Under the assumption that all the sources in the HGPS catalogue with flux $\Phi \geq 0.1 \Phi_{\mathrm{CRAB}}$ (except those firmly identified as SNRs) are powered by pulsar activity and assuming a constant source formation rate $R$ we get $\tau_{s d}=1.8_{-0.6}^{+1.5} \times 10^{3} \mathrm{yr}$ for our reference case.

The value for the spin-down time scale can be used to determine, through Eqs.(10), the initial period $P_{0}$ and magnetic field $B_{0}$ of the pulsar-powered population. We get the constraints $P_{0}=33.5_{-4.3}^{+5.4} \mathrm{~ms} \times\left(\lambda / 10^{-3}\right)^{1 / 2}$ and $B_{0}=4.3(1 \pm 0.45) 10^{12} \mathrm{G} \times\left(\lambda / 10^{-3}\right)^{1 / 2}$ that correspond to the purple dot dashed line and the green dotted line respectively in the right panel of Fig. 2. The inferred magnetic field agrees with the value $\log _{10}\left(B_{0} / 1 G\right) \simeq 12.65$ obtained by pulsar population studies [7]. The inferred period is consistent with the value $P_{0} \sim 50 \mathrm{~ms}$ obtained in [13] by studying $\gamma$-ray pulsar population while is in tension with the value $P_{0} \sim 300 \mathrm{~ms}$ that is obtained from pulsar radio observation [7] (unless one assumes $\lambda \sim 10^{-1}$ ). A conservative upper bound for the period $P_{0}$ can be obtained by considering that no less than 10 of these sources have to be necessarily included in this population, being firmly identified as PWNe or Composite Sources. The lines $N\left(0.1 \Phi_{\mathrm{CRAB}}\right)=$ const, corresponding to a fixed number of sources above the adopted flux threshold $0.1 \Phi_{\mathrm{CRAB}}$, are shown with gray dashed lines in the planes $\left(L_{\max }, \tau\right)$ and $\left(P_{0}, B_{0}\right)$ in 

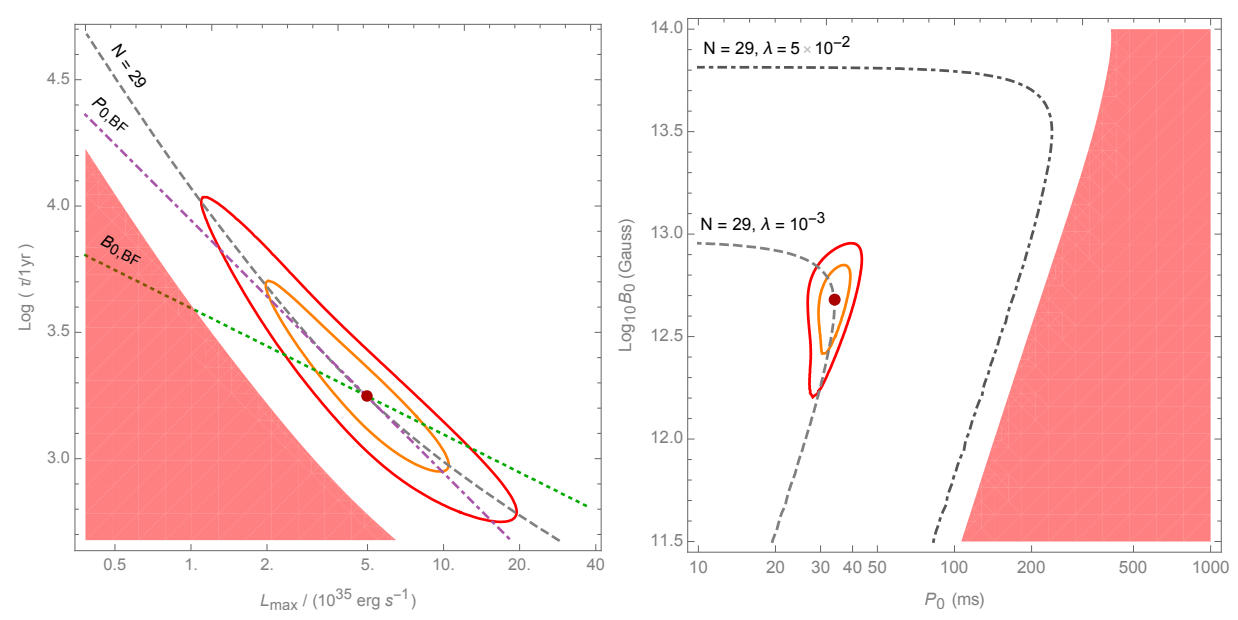

Figure 2: Left Panel: The best fit and the $1 \sigma$ and $2 \sigma$ allowed regions in the plane $\left(L_{\max }, \tau\right)$. The red shaded area is excluded by the data because corresponds to $N\left(0.1 \Phi_{\mathrm{CRAB}}\right) \leq 10$ Right Panel: The best fit and the $1 \sigma$ and $2 \sigma$ allowed regions in the plane $\left(P_{0}, B_{0}\right)$, calculated in the assumption that $\lambda=10^{-3}$. The red shaded area corresponds to $N\left(0.1 \Phi_{\mathrm{CRAB}}\right) \leq 10$ in the assumption of $\lambda=5 \times 10^{-2}$.

Fig. 2. It can be demonstrated analytically (see Appendix in [5]) that the condition $N(\Phi)=$ const always individuates a maximum allowed period $P_{0}$ whose specific value depends on the fraction $\lambda$ of the pulsar spin-down energy that is converted to $\mathrm{TeV} \gamma$-ray emission. In particular, the red shaded area in Fig. 2 can be excluded because it corresponds to $N\left(0.1 \Phi_{\mathrm{CRAB}}\right) \leq 10$ and to the relatively large value $\lambda=5 \times 10^{-2}$. This allows us to obtain the bound $P_{0} \leq 500 \mathrm{~ms}$ that can be strengthened if an upper limit for the magnetic field $B_{0} \leq 10^{14} \mathrm{G}$ is introduced.

The fact that the inferred values for $B_{0}$ and $P_{0}$ are consistent with expectations justifies the working assumption that a large fraction of bright sources observed by H.E.S.S. belongs to a population of young pulsars, and supports the hypothesis, formulated e.g. by [9] and [12], that $\mathrm{PWNe}$ and/or TeV halos could produce the majority of TeV bright sources in the Sky.

\section{Conclusions}

In this work, we present a novel analysis of the flux, longitude and latitude distributions of the brightest sources ( $\Phi \geq 10 \% \Phi_{\mathrm{CRAB}}$ ) of the HGPS catalogue showing that the luminosity distribution of Galactic $\mathrm{TeV}$ sources can be effectively constrained.

More precisely, by assuming that the luminosity function is described by a power-law (see Eq. (2) we extract the source maximal luminosity $L_{\max }$ and the high-luminosity normalization of the source distribution $\mathcal{N}$ (the spin-down timescale $\tau_{\text {sd }}$ ) by fitting HPGS data. This allows us to determine the total Milky Way luminosity $L_{\mathrm{MW}}$ in the energy range $1-100 \mathrm{TeV}$ and the total Galactic flux in the H.E.S.S. OW given by $\Phi_{\text {tot }}$. In addition, the total source flux is relatively large, implying that unresolved source contribution is not negligible (about $60 \%$ of the resolved signal measured by H.E.S.S.) and potentially responsible for a large fraction of the diffuse large-scale gamma-ray signal observed by H.E.S.S.. This could have important implications for the interpretation of current observations of other experiments in the $\mathrm{TeV}$ domain. 
Moreover, considering the possibility that the bright sources observed by H.E.S.S. are powered by pulsar activity, we evaluate the constraints on the physical properties of the pulsar population. For our reference case, assuming that the fraction of the pulsar spin-down energy converted in $\mathrm{TeV}$ photons is $\lambda=10^{-3}$, we obtain the best-fit values for the initial spin-down period $P_{0}$ and the magnetic field $B_{0}$. The above constraints are consistent with the $B_{0}$ value obtained in [7] and $P_{0}$ constrains described in [13] by studying the gamma-ray pulsar population.

\section{Acknowledgments}

This work was partially supported by the research grant number 2017W4HA7S "NAT-NET: Neutrino and Astroparticle Theory Network" under the program PRIN 2017 funded by the Italian Ministero dell'Istruzione, dell'Universita' e della Ricerca (MIUR).

\section{References}

[1] Abdalla, H., Abramowski, A., Aharonian, F., et al. 2018, Astron. Astrophys., 612, A1, doi: $10.1051 / 0004-6361 / 201732098$

[2] —. 2018, Astron. Astrophys., 612, A2, doi: 10.1051/0004-6361/201629377

[3] Abeysekara, A. U., Albert, A., Alfaro, R., et al. 2017, Astrophys. J., 843, 40, doi: 10 . 3847/ $1538-4357 /$ aa7556

[4] Cataldo, M., Pagliaroli, G., Vecchiotti, V., \& Villante, F. 2019, JCAP, 12, 050, doi: 10 . 1088/ $1475-7516 / 2019 / 12 / 050$

[5] Cataldo, M., Pagliaroli, G., Vecchiotti, V., \& Villante, F. L. 2020, Astrophys. J., 904, 85, doi: $10.3847 / 1538-4357 /$ abc0ee

[6] Diehl, R., Halloin, H., Kretschmer, K., et al. 2006, Nature, 439, 45, doi: 10.1038/ nature 04364

[7] Faucher-Giguere, C.-A., \& Kaspi, V. M. 2006, Astrophys. J., 643, 332, doi: 10 . 1086/501516

[8] Gaensler, B. M., \& Slane, P. O. 2006, Ann. Rev. Astron. Astrophys., 44, 17, doi: 10.1146/ annurev. astro.44.051905.092528

[9] Linden, T., \& Buckman, B. J. 2018, Phys. Rev. Lett., 120, 121101, doi: 10.1103/ PhysRevLett. 120.121101

[10] Lorimer, D. R., Faulkner, A. J., Lyne, A. G., et al. 2006, Mon. Not. Roy. Astron. Soc., 372, 777, doi: $10.1111 / \mathrm{j} .1365-2966.2006 .10887 . \mathrm{x}$

[11] Strong, A. W. 2007, Astrophys. Space Sci., 309, 35, doi: 10. 1007/s10509-007-9480-1

[12] Sudoh, T., Linden, T., \& Beacom, J. F. 2019, Phys. Rev. D, 100, 043016, doi: 10.1103/ PhysRevD.100.043016

[13] Watters, K. P., \& Romani, R. W. 2011, Astrophys. J., 727, 123, doi: 10.1088/0004-637X/ $727 / 2 / 123$ 